\title{
Role of Intermediate Care Unit Admission and Noninvasive Respiratory Support during the COVID-19 Pandemic: A Retrospective Cohort Study
}

\author{
Olivier Grosgurin ${ }^{a} \quad$ Antonio Leidia Pauline Darbellay Farhoumand ${ }^{a}$ \\ Sebastian Carballo ${ }^{a}$ Dan Adler ${ }^{c}$ Jean-Luc Reny ${ }^{a}$ Bernardo Bollen Pinto ${ }^{b}$ \\ Anne Rossel $^{a}$ Jacques Serratrice ${ }^{a}$ Thomas Agoritsas $^{a}{ }^{d}$ Jérôme Stirnemann ${ }^{a}$ \\ Christophe Marti ${ }^{a}$ \\ aDepartment of Medicine, Division of General Internal Medicine, Geneva University Hospitals, Geneva, Switzerland; \\ ${ }^{b}$ Department of Acute Medicine, Division of Anesthesiology, Geneva University Hospitals, Geneva, Switzerland; \\ 'Department of Medicine, Division of Lung diseases, Geneva University Hospitals, Geneva, Switzerland; ${ }^{\mathrm{d} D e p a r t m e n t}$ of \\ Health Research Methods, Evidence, and Impact, Hamilton, ON, Canada
}

\section{Keywords}

Noninvasive ventilation - Respiratory insufficiency .

Intermediate care unit

\begin{abstract}
Background: The COVID-19 pandemic has led to shortage of intensive care unit (ICU) capacity. We developed a triage strategy including noninvasive respiratory support and admission to the intermediate care unit (IMCU). ICU admission was restricted to patients requiring invasive ventilation. $\mathbf{O b}$ jectives: The aim of this study is to describe the characteristics and outcomes of patients admitted to the IMCU. Method: Retrospective cohort including consecutive patients admitted between March 28 and April 27, 2020. The primary outcome was the proportion of patients with severe hypoxemic respiratory failure avoiding ICU admission. Secondary outcomes included the rate of emergency intubation, 28day mortality, and predictors of ICU admission. Results: One hundred fifty-seven patients with COVID-19-associated pneumonia were admitted to the IMCU. Among the 85 patients admitted for worsening respiratory failure, 52/85 (61\%) avoided ICU admission. In multivariate analysis, $\mathrm{PaO}_{2} /$
\end{abstract}

karger@karger.com

(c) 2021 S. Karger AG, Basel

www.karger.com/res

Karger"
$\mathrm{FiO}_{2}$ (OR 0.98; 95\% Cl: 0.96-0.99) and BMI (OR 0.88; 95\% Cl: 0.78-0.98) were significantly associated with ICU admission. No death or emergency intubation occurred in the IMCU. Conclusions: IMCU admission including standardized triage criteria, self-proning, and noninvasive respiratory support prevents ICU admission for a large proportion of patients with COVID-19 hypoxemic respiratory failure. In the context of the COVID-19 pandemic, IMCUs may play an important role in preserving ICU capacity by avoiding ICU admission for patients with worsening respiratory failure and allowing early discharge of ICU patients.

(c) 2021 S. Karger AG, Basel

\section{Introduction}

The widespread expansion of the coronavirus disease 2019 (COVID-19) has led to a global pandemic and acute rise in hospital and intensive care unit (ICU) admissions [1]. Many countries experienced capacity shortage in acute care and ICU, due to the sharp increase in admission of patients with acute hypoxemic respiratory failure (AHRF) and the prolonged length of ICU stay for pa-

Correspondence to:

Olivier Grosgurin, olivier.grosgurin@ @cuge.ch 
tients requiring invasive mechanical ventilation [2-4]. At the peak of the pandemic, pessimistic projections predicted $>3$ million ICU admissions in the USA and $>30$ potential critically ill patients per ventilator place $[3,5]$. Alarmingly poor outcomes were reported from Wuhan among patients requiring invasive mechanical ventilation, with mortality rates ranging between 81 and $97 \%$ [6]. The COVID-19 pandemic compelled health authorities of several countries to establish triage committees and restrictive admission criteria for ICU resource allocation $[5,7]$.

Intermediate care units (IMCUs), also known as high-dependency units, typically stand between regular ward and ICU. In Switzerland, these units provide continuous monitoring and an increased nurse-to-patient ratio compared to standard wards [8]. These units are able to provide several modalities of noninvasive respiratory support or vasopressors while patients requiring invasive mechanical ventilation are admitted to the ICU.

In the context of COVID-19 respiratory failure, IMCU may be the ideal structure to evaluate patient's responsiveness to noninvasive respiratory support such as continuous positive airway pressure (CPAP) and high-flow nasal oxygen (HFNO) and to restrict ICU admission for those who fail to respond to noninvasive treatment. In addition, early discharge to the IMCU of ICU patients still requiring monitoring, heavy burden of care, or specific procedures such as ventilation or tracheotomy weaning may contribute to preserve ICU capacity [9].

Our objective was to evaluate the contribution of IMCUs in the management of the COVID-19 respiratory pandemic. The primary objective was to evaluate the proportion of patients with severe hypoxemic respiratory failure admitted to the IMCU avoiding ICU admission and invasive mechanical ventilation.

Our secondary objectives were to assess the safety of this strategy by recording unexpected emergency intubation outside the ICU, mortality at 28 days, and the occurrence of sick leaves among IMCU healthcare workers due to COVID-19 infections. We also assessed the role of transfer to the IMCU as a step-down unit after ICU stay, in order to preserve ICU capacity.

\section{Methods}

The study protocol was written according the STROBE Statement Checklist and is available on request (https://www.equatornetwork.org/). This observational study was approved by the ethics committee of our institution (CCER 2020-01087).

Noninvasive Respiratory Support for COVID-19
Study Design and Setting

We performed a retrospective cohort study of patients admitted to the IMCU of Geneva University Hospitals (HUG), Switzerland, during the COVID-19 pandemic. Consecutive patients admitted between March 28 and April 27, 2020, were included. In order to absorb the high volume of admissions, our IMCU capacity was progressively raised from 9 to 42 beds for patients with COVID-19 during the observation period. The capacity of the medical IMCU was increased by first transforming our coronary care and stroke units into medical IMCUs. Then, a new floor of IMC including 30 beds was established in collaboration with the Division of Anesthesiology. Additional Hamilton ${ }^{\circledR}$ T1 ventilators were provided by the Swiss Army forces. Nurses from the divisions of internal medicine and anesthesiology were attributed to this extended unit, as well as staff available after the stopping of surgical activity during the pandemic. The nurse-to-patient ratio was $2-5$ with a supervising nurse (IMCU or anesthesiology trained nurse) for every 10 patients. As a comparison, the nurse-to-patient ratio is 1-8 during daytime in the ward units and 1-2 in the ICU with at least $1 \mathrm{ICU}$-certified nurse in each module of 10 patients. A dedicated respiratory therapist was available for the IMCU around the clock. For every 15 IMCU beds, the physician staff was the following: 2 residents and 1 senior internal medicine or anesthesiology resident. This medical staff was equivalent to the one in the ICU and superior to the one in the ward ( 3 internal residents and 1 senior resident for 26 beds).

\section{Participants}

Inclusion Criteria

Patients were included in case of COVID-19 pulmonary disease confirmed by SARS-Cov-2 reverse transcriptase polymerase chain reaction performed on either nasopharyngeal or oropharyngeal swabs and image of pulmonary infiltrate and admission to IMCUs between March 28 and April 27, 2020.

\section{Exclusion Criteria}

Patients were excluded in case of IMCU admission for reasons other than COVID-19 pulmonary disease. In addition, patients for which admission to the ICU was no more considered after decision of treatment de-escalation were excluded from the univariate and multivariate models exploring the likelihood of ICU admission.

\section{Procedures}

In our institution, admission criteria to the IMCU were defined as the need for an inspired fraction of oxygen $\left(\mathrm{FiO}_{2}\right)$ superior to $50 \%$ to maintain an oxygen saturation superior to $90 \%$ and the absence of signs of respiratory distress (defined as use of accessory muscles, intercostal retraction, or alteration of mental status). Admission criteria to the IMCU and ICU during the study period are provided in Table 1.

HFNO was delivered using the Optiflow ${ }^{\circledR}$ system and Hamilton ${ }^{\circledR} \mathrm{T} 1$ ventilators with a humidifier. In order to limit potential aerosolization hazards, oxygen flow was set to $30 \mathrm{~L} / \mathrm{min}$, and higher flows $(50 \mathrm{~L} / \mathrm{min})$ were allowed for patients showing improvement with CPAP but unable to tolerate it. $\mathrm{FiO}_{2}$ was titrated according to oxygen saturation aiming a saturation comprised between 90 and $94 \%$.

CPAP was delivered during 6 sessions lasting for $2 \mathrm{~h}$ through full face masks aiming for a positive end-expiratory pressure value of $10 \mathrm{~cm} \mathrm{H}_{2} \mathrm{O}$ alternating with HFNO. An expiratory filter was 
Table 1. IMCU and ICU admission criteria at the University Hospital of Geneva during the COVID-19 pandemic*

\begin{tabular}{ll}
\hline Criteria for IMCU & Criteria for ICU \\
\hline $\mathrm{SaO}_{2}<90 \%$ despite $\mathrm{FiO}_{2}>50 \%$ and no respiratory distress & $\begin{array}{l}\text { Admission from medical wards } \\
\mathrm{SaO}_{2}<90 \% \text { despite } \mathrm{FiO}_{2}>50 \% \text { and respiratory distress }\end{array}$ \\
\cline { 2 - 2 } & $\begin{array}{l}\mathrm{Admission} \text { from the } \mathrm{IMCU} \\
\mathrm{SaO}_{2}<90 \% \text { despite } \mathrm{FiO}_{2}>80 \% \text { with HFNO and respiratory distress }\end{array}$ \\
\hline
\end{tabular}

IMCU, intermediate care unit; ICU, intensive care unit; HFNO, high-flow nasal oxygen. *No therapeutic limitation regarding orotracheal intubation.

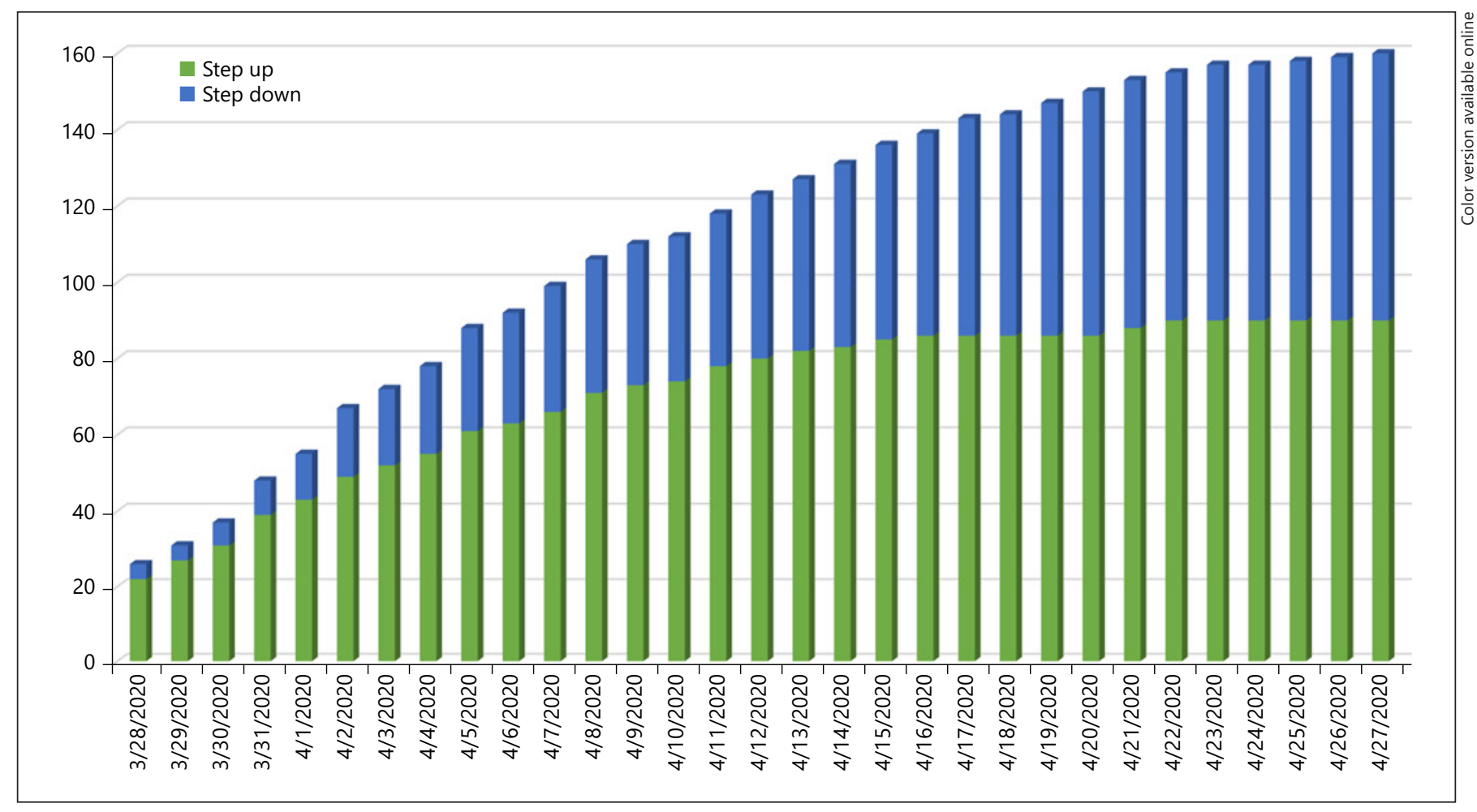

Fig. 1. Cumulative number of "step-up" and "step-down" patients in the IMCU. IMCU, intermediate care unit.

systematically used. Using awake prone position was left at the discretion of the treating physician and repeated according to tolerance and improvement in oxygenation parameters. Protective personal equipment (glasses, FFP2 masks, gloves, and protective overdresses) was mandatory in the entire zone of care in the IMCU and ICU.

\section{Variables}

Demographic (age and gender) variables, comorbidities, BMI, respiratory parameters $\left(\mathrm{FiO}_{2}, \mathrm{PaO}_{2} / \mathrm{FiO}_{2}\right.$, respiratory rate, and $\mathrm{PaCO}_{2}$ at admission), heart rate, symptoms duration, IMCU length of stay, and receipt of antiviral therapy were extracted from the medical reports. Living status at 28 days was established based on medical records, administrative database of the Geneva prov- ince, or contact with patients or relatives. The total number of cases hospitalized in our institution was extracted from our administrative database.

\section{Data Sources}

The main data source was the electronic patient data system $\left(\mathrm{DPI}^{\circledR}\right.$ ) of the Geneva University Hospital. Data regarding the total number of patients admitted to the hospital and patients' trajectories from the ward or emergency department to the ICU were obtained from the institutional administrative database. Data regarding the prevalence of absences due to COVID-19 infection among IMCU health workers were obtained from our administrative database (Vision $\mathrm{RH}^{\circledR}$ ) and included nurses and auxiliaries affiliated to these units during the study period. 
Table 2. Patient characteristics of IMCU step-up admissions according to the need for subsequent ICU transfer

\begin{tabular}{|c|c|c|c|c|}
\hline & All patients $(n=85)$ & ICU transfer $(n=33)$ & No ICU transfer $(n=52)$ & $p$ value \\
\hline Age, median (IQR), years & $64.0(56.0,74.5)$ & $66.0(57.5,76.5)$ & $62.0(54.3,74.0)$ & 0.38 \\
\hline Male, $n(\%)$ & $64(75.3)$ & $28(84.8)$ & $36(69.2)$ & 0.10 \\
\hline Comorbidities, any, $n$ (\%) & $53(61.6)$ & $21(63.6)$ & $31(59.6)$ & 0.71 \\
\hline Cancer, $n(\%)$ & $5(5.9)$ & $2(6.1)$ & $3(3.5)$ & 0.96 \\
\hline Hypertension, $n(\%)$ & $37(43.5)$ & $14(16.5)$ & $23(27.1)$ & 0.87 \\
\hline Cardiac disease, $n(\%)$ & $19(22.4)$ & $11(33)$ & $8(15.4)$ & 0.05 \\
\hline COPD, $n(\%)$ & $5(5.9)$ & $3(3.5)$ & $2(2.4)$ & 0.32 \\
\hline Renal failure, $n(\%)$ & $10(11.8)$ & $3(3.5)$ & $7(8.2)$ & 0.54 \\
\hline BMI, median (IQR), $\mathrm{kg} / \mathrm{m}^{2}$ & $26.7(23.8,31.9)$ & $25.7(23.5,30.6)$ & $27.6(24.1,32.4)$ & 0.187 \\
\hline $\mathrm{FiO}_{2}$, median (IQR) & $0.62(0.5,0.7)$ & $0.65(0.58,0.80)$ & $0.60(0.41,0.65)$ & 0.01 \\
\hline $\mathrm{PaO}_{2} / \mathrm{FiO}_{2}$, median (IQR), $\mathrm{mm} \mathrm{Hg}$ & $133.6(94.5,166.5)$ & $106.5(75.9,142.9)$ & $140.6(106.3,183.6)$ & 0.01 \\
\hline $\mathrm{PaCO}_{2}$, median (IQR), mm Hg & $33.4(30.2,37.7)$ & $32.9(28.8,37.7)$ & $33.9(31.5,38.0)$ & 0.71 \\
\hline Symptom duration, median (IQR) & $9.0(6.0,13.0)$ & $8.0(6.5,12.0)$ & $10.0(6.0,14.0)$ & 0.47 \\
\hline IMCU LOS, median (IQR), days & $3.0(1.0,6.0)$ & $1.0(0.0,1.0)$ & $3.0(2.0,6.75)$ & $<0.001$ \\
\hline 28-day mortality, $n(\%)$ & $10(11.8)$ & $5(15.2)$ & $5(9.6)$ & 0.44 \\
\hline
\end{tabular}

IQR, interquartile range; IMCU, intermediate care unit; ICU, intensive care unit; LOS, length of stay.

\section{Outcomes}

The primary outcome was the proportion of patients admitted to the ICU among patients admitted to the IMCU for worsening hypoxemia (i.e., patients admitted in a step-up strategy). Secondary outcomes included 28-day mortality, the number of deaths or emergent intubation in the IMCU, length of IMCU stay, and absences due to COVID-19 infections among healthcare workers. COVID-19-related absences occurring from March 28 until May 10, 2020, were included in order to account for a 2 -week incubation period.

\section{Statistical Methods}

The collection of data was performed by a research nurse ( $\mathrm{T}$. Mann), a biologist (L. Bayer), and 2 investigators (A.L. and O.G.). Our study had a convenient sample size, and no sample calculation was performed. Patients admitted to the IMCU of Geneva Hospitals since March 27 were included as IMCU admission criteria for COVID-19 were established at this time. Recruitment was stopped on April 28 because the rate of admission of COVID-19 patients declined in our institution during the last week of this month and to allow us to complete 28-day follow-up at the end of May 2020.

\section{Statistical Analysis}

Demographic and descriptive statistics are provided in medians with interquartile ranges (IQR). Categorical and continuous variables were measured and compared between subsets of patients using $\chi^{2}$ and nonparametric tests as appropriate. $p$ values for between-group differences are provided with a statistical significance level defined as $p$ value $<0.05$. In order to identify predictors of ICU admission, association between dependent variables and the primary outcome was assessed using univariate and multivariate logistic regression. The multivariate model was built using 5 predefined clinical variables (age, $\mathrm{BMI}$, respiratory rate, heart rate, and $\left.\mathrm{PaO}_{2} / \mathrm{FiO}_{2}\right)$. Analyses were conducted with IBM SPSS Statistics software (version 25) except for the multivariate model, for which R statistical software ( $\mathrm{R}$ foundation) was used.

\section{Results}

During the study period, 2,607 COVID infections were diagnosed in the Geneva province, 671 COVID patients were admitted to our hospital, and 63 were admitted to the ICU. One hundred fifty-seven patients were admitted to the IMCU with COVID-associated pneumonia including 85 patients with worsening hypoxemic respiratory failure (step-up admissions) and 72 patients discharged from the ICU to the IMCU (step-down admissions) (Fig. 1). The characteristics of patients admitted to the IMCU for treatment intensification are provided in Table 2. Sixty-four out of 85 patients (75.3\%) were male with a median age of 64.0 years (IQR 56.0, 74.5). The median arterial partial pressure of oxygen/ fraction of inspired oxygen ratio $\left(\mathrm{PaO}_{2} / \mathrm{FiO}_{2}\right)$ at IMCU admission was $133.6 \mathrm{~mm} \mathrm{Hg}$ (IQR 94.5, 166.5), and median BMI was $26.7 \mathrm{~kg} / \mathrm{m}^{2}$ (IQR 23.8, 31). Nineteen patients $(22.4 \%)$ were admitted from the Emergency Department and $66(77.6 \%)$ from the ward. 
Respiratory support aiming at 6 sessions of $2 \mathrm{~h}$ of CPAP per day and continuous HFNO between them was prescribed to all 85 patients admitted for worsening hypoxemia as part of our local treatment protocol. Unfortunately, duration of this respiratory support was not precisely documented during the beginning of the pandemic. Of the 85 patients admitted for worsening hypoxemia (step-up admissions), 33 (39\%) required ICU admission and invasive mechanical ventilation and 52 (61\%) were discharged to the ward without being admitted to the ICU.

Compared to patients discharged to the ward, patients requiring ICU admission required higher $\mathrm{FiO}_{2}$ at admission and had lower $\mathrm{PaO}_{2} / \mathrm{FiO}_{2}$ ratios and higher respiratory rates (Table 2). The median IMCU length of stay was 1.0 day (IQR 0,1$)$ for patients requiring ICU admission and 3.0 days (IQR 2.0, 6.75) for those transferred to the ward. No unexpected death or emergent intubation occurred in the IMCU. The 28-day mortality was $15.2 \%$ (5/33) among patients requiring ICU admission and 9.6\% $(5 / 52)$ for patients transferred to the ward. The $5 \mathrm{IMCU}$ patients who died without being admitted to the ICU died in the ward after decision of treatment de-escalation due to unfavorable course and discussion with the patient and/or relatives.

In univariate analysis, respiratory rate at IMCU admission (OR 1.16; 95\% CI: 1.06-1.31) and reduced $\mathrm{PaO}_{2} /$ $\mathrm{FiO}_{2}$ ratio (OR 0.98; 95\% CI: 0.97-0.99) were significantly associated with ICU admission. In the multivariate model including age, BMI, heart rate, respiratory rate, SOFA score, and $\mathrm{PaO}_{2} / \mathrm{FiO}_{2}, \mathrm{PaO}_{2} / \mathrm{FiO}_{2}$ (OR 0.98, 95\% CI: 0.96-0.99) and BMI (OR 0.87; 95\% CI: 0.76-0.97) were significantly associated with ICU admission (Table 3).

From March 28 to May 15, the COVID-19-related absence rate among healthcare workers was $4.9 \%(5 / 103)$ in the IMCU, $1.6 \%(4 / 249)$ in the ICU, and $12.5 \%(50 / 401)$ in the wards. The rate of COVID-19 absence was significantly lower in the IMCU compared to the wards (risk difference $7.6 \%$; 95\% CI: $0.92-12.15, p=0.03$ ). No statistically significant difference was observed between IMCU and ICU (risk difference 3.3\%; 95\% CI: 0.4-9.4, $p=0.08$ ).

\section{Discussion}

Our study suggests that IMCU admission may contribute to prevent ICU admission and invasive mechanical ventilation in a majority of patients with COVID19-associated AHRF without signs of respiratory distress.
Table 3. Association between patient characteristics and transfer to the ICU among IMCU step-up admissions

\begin{tabular}{lll}
\hline Variable & $\begin{array}{l}\text { Univariate OR } \\
(95 \% \mathrm{CI})\end{array}$ & Multivariate OR \\
\hline Age & $1.01(0.98-1.05)$ & $1.03(0.98-1.09)$ \\
$\mathrm{BMI}, \mathrm{kg} / \mathrm{m}^{2}$ & $0.94(0.85-1.02)$ & $0.87(0.76-0.97)$ \\
$\mathrm{Heart} \mathrm{rate}_{\mathrm{min}} \mathrm{m}^{-1}$ & $1.01(0.99-1.04)$ & $1.00(0.97-1.03)$ \\
Respiratory rate $_{\mathrm{PaO}_{2} / \mathrm{FiO}_{2}, \mathrm{~mm} \mathrm{Hg}}$ & $1.16(1.06-1.31)$ & $1.04(0.90-1.20)$ \\
$\mathrm{SOFA} \mathrm{score}$ & $0.98(0.97-0.99)$ & $0.98(0.96-0.99)$ \\
\hline
\end{tabular}

IMCU, intermediate care unit; ICU, intensive care unit.

Despite severe hypoxemia (median $\mathrm{PaO}_{2} / \mathrm{FiO}_{2} 133.6 \mathrm{~mm}$ $\mathrm{Hg}$ ), about $60 \%$ of patients in our cohort were transferred back to the ward without the need for an ICU admission. This finding may be highly relevant in the context of a respiratory pandemic where ICU capacity is a critical issue. However, these findings must be interpreted with caution given the observational design of our study and the absence of a control group. Various factors including standardized admission criteria, increased nurse-to-patient ratio, self-proning, staff expertise, and noninvasive respiratory support may actually contribute to the favorable outcome of the majority of IMCU patients.

The role of noninvasive ventilation in AHRF has been debated over the last decades as delaying intubation for patients requiring invasive mechanical ventilation has been reported to be associated with negative patient outcome [10]. Moreover, noninvasive ventilation with inspiratory aid may be associated with concern regarding the risk of volutrauma in patients with spontaneous breathing $[11,12]$. HFNO has been reported to reduce 90 -day mortality in a randomized control trial including $310 \mathrm{pa}-$ tients with AHRF compared to standard oxygen or noninvasive ventilation [13]. In a recent network meta-analysis including 25 studies and 3,804 patients, both noninvasive ventilation and $\mathrm{HFNO}$ reduced the rate of orotracheal intubation among patients with AHRF [14]. The network effect estimates suggested that helmet noninvasive ventilation might be the most effective option through allowing higher levels of positive end-expiratory pressure and reducing inspiratory efforts $[15,16]$. However, this evidence relies mainly on indirect comparisons and on studies including a large proportion of patients with community-acquired pneumonia or immunosuppression. These patients may differ from COVID-19-associated AHRF. Indeed, different phenotypes of acute 
lung injury have been reported among patients with $\mathrm{CO}-$ VID-19-associated AHRF including near preserved lung compliance and low ventilation-to-perfusion ratios [17]. In our cohort, mortality of patients transferred to the ICU from the IMCU was $15.2 \%$ compared with $19.1 \%$ among the entire ICU cohort at our institution [18].

In our institution, we decided to combine HFNO using nasal cannulas and CPAP delivered through a facial mask for pragmatic reasons. These 2 modalities were possible using the same ventilator (Hamilton ${ }^{\circledR} \mathrm{T} 1$ ), and our IMCU nurses and respiratory therapists are more used to this interface. Because some concern has been raised regarding the risk of potential aerosolization and healthcare workers contamination during HFNO or noninvasive ventilation, we aimed to reduce this risk by using personal protective equipment including glasses, FFP2 masks, gloves, and protective overdresses in the entire zone of care. Moreover, oxygen flow was restricted to $30 \mathrm{~L} / \mathrm{min}$ using HFNO, except for patients with significant improvement during CPAP but unable to tolerate the mask interface. Using these precautions, the rate of contamination among IMCU healthcare workers in our study (4.9\%) was low and similar to the rate of infection in the Geneva province according to a large seroprevalence study conducted at our institution [19]. In comparison, the rate of COVID-related absence during the study period was $12.5 \%(50 / 401)$ in the wards and $1.6 \%(4 / 249)$ in the ICU in our institution. The higher rate of healthcare workers infection in the wards compared to ICU and IMCU may be partly due to the mandatory use of protective personal equipment including FFP2 masks in the ICU and IMCU but not in the wards where FFP2 masks were recommended only for specific procedures. However, our study only considered symptomatic infections leading to the absence of collaborators and could therefore fail to identify asymptomatic infections. Moreover, the potential source of contamination was not investigated.

Other emerging evidence regarding the role of noninvasive respiratory support in the context of COVID-19 AHRF has recently been reported. In a retrospective study comparing 2 consecutive periods, Oranger et al. [20] reported a rate reduction of invasive mechanical ventilation in the intervention period using CPAP compared to the control period using increasing supplemental oxygen. Similarly, HFNO or CPAP combined with patient selfproning has been reported to improve oxygenation in several case series [21-23].

In our cohort, combining HFNO, CPAP, and patient self-proning in an IMCU resulted in a high proportion of patients avoiding invasive mechanical ventilation in the
ICU. A key consideration is the use of standardized criteria for IMCU admission based on the absence of signs of respiratory distress. This strategy is in accordance with the World Health Organization guidance for the use of HFNO in COVID-19-associated AHRF recommending against the use of HFNO for patients with hypercapnia, hemodynamic instability, multiorgan failure, or abnormal mental status [24].

As previously reported by others, being overweight was highly prevalent among patients developing AHRF requiring admission to our IMCU [25]. Unexpectedly, increasing BMI was inversely associated with ICU admission in our cohort. This apparent paradox may suggest that overweight patients might respond more favorably to noninvasive respiratory support than patients with other mechanisms of AHRF. This observation may also be explained by the fact that the median BMI in our cohort was in the "overweight" range rather than overt obesity. Previous studies reported a J-shaped association between BMI and mortality [26]. Therefore, the inverse association between mortality and BMI may not be true among populations in the overt obesity range.

During the study period, about one-half of patients admitted to the ICU were admitted from the IMCU, the remaining being admitted from the ED (21/63) or directly from the ward (9/63), suggesting that these criteria may be applied to an important proportion of patients. These empirically established criteria were based on the observation that a subset of patients with COVID-19 AHRF showed an excellent tolerance to hypoxemia (sometimes referred to as "happy hypoxemia").

Finally, avoiding invasive mechanical ventilation in the context of the COVID-19 pandemic does not only preserve ICU capacity, but may also benefit patients by preventing invasive mechanical ventilation-related complications. In particular, the prevalence of neurological complications was high among ICU survivors discharged to our IMCU compared to IMCU patients transferred to the ward. This difference may be partly explained by the selection of patients with increased COVID-19 disease severity to be transferred to the ICU and the fact that the occurrence of obtundation or altered mental status may have prompted ICU admission in a subset of patients. However, the high prevalence of muscle weakness, ICUacquired polyneuropathy, and delirium among ICU survivors may also result of the need for prolonged sedation, use of paralyzing agents, and invasive mechanical ventilation.

Our study has several limitations. First, we used a convenient sample size, limiting the number of events and 
our ability to exhaustively identify predictors of ICU admission. Moreover, several variables of our model were part of the decision to provide invasive ventilation which can lead to incorporation bias. Second, IMCUs do not exist worldwide, precluding the generalizability of our observation. However, we believe that our experience could be an interesting model in other settings and may apply to similar structures such as respiratory care or high-dependency units. Third, we used a combination of HFNO, CPAP, and self-prone position. The duration of interventions was not systematically recorded, preventing us to analyze the benefit of each modality individually. Finally, our observational design precludes drawing definite conclusion about the beneficial effect of IMCU admission or noninvasive ventilation in the absence of a control group. Further research, including interventional controlled studies, is needed to confirm our findings.

\section{Conclusion}

Our IMCU admitted patients with severe COVID-19 AHRF yet without respiratory distress allowing to prevent a large proportion of patients from being transferred to the ICU. This model of care may be beneficial to preserve ICU capacity and reduce the complications associated with invasive mechanical ventilation.

\section{Acknowledgements}

We thank our study nurses, Tamara Mann and Laurence Bayer, for data collection, and Prof. Bernhard Walder for his advice and clinical collaboration. We would like to thank sincerely all nurses, residents, and other healthcare providers who allowed us to manage the COVID pandemic at the IMCU of the Geneva University Hospital, as well as the heads of the Departments of Medicine and Acute Care for their trust and support during this challenging period.

\section{Statement of Ethics}

This study was conducted ethically in accordance with the World Medical Association Declaration of Helsinki. It was approved by the ethics committee of our institution (CCER 202001087).

\section{Conflict of Interest Statement}

The authors have no conflicts of interest to declare.

\section{Funding Sources}

This study had no financial support.

\section{Author Contributions}

O.G., C.M., and A.L. participated in data acquisition, design, and drafting of the manuscript. O.G. and J.S.T. performed the statistical analyses. All authors participated in study conception, data interpretation, and critical revision of the manuscript for important intellectual content. All authors approved the version submitted for publication and agreed to be accountable regarding accuracy and integrity of any part of the work. C.M. and O.G. had full access to the data and take responsibility for the integrity and the accuracy of the data.

\section{References}

1 Li R, Rivers C, Tan Q, Murray MB, Toner E, Lipsitch M. Estimated demand for US hospital inpatient and intensive care unit beds for patients with COVID-19 based on comparisons with Wuhan and Guangzhou, China. JAMA Netw Open. 2020 May 1;3(5):e208297.

2 Carenzo L, Costantini E, Greco M, Barra FL, Rendiniello V, Mainetti M, et al. Hospital surge capacity in a tertiary emergency referral centre during the COVID-19 outbreak in Italy. Anaesthesia. 2020 Jul;75(7):928-34.

3 Emanuel EJ, Persad G, Upshur R, Thome B, Parker M, Glickman A, et al. Fair allocation of scarce medical resources in the time of Covid-19. N Engl J Med. 2020 May;382(21): 2049-55.

4 Ramachandran P, Swamy L, Kaul V, Agrawal A. A national strategy for ventilator and ICU resource allocation during the COVID-19 pandemic. Chest. 2020 Sep;158(3):887-9.

5 Truog RD, Mitchell C, Daley GQ. The toughest triage: allocating ventilators in a pandemic. N Engl J Med. 2020 May 21;382(21):19735.

6 Weiss P, Murdoch DR. Clinical course and mortality risk of severe COVID-19. Lancet. 2020 Mar 28;395(10229):1014-5.

7 Swiss Academy of Medical Sciences. COVID-19 pandemic: triage for intensive-care treatment under resource scarcity. Swiss Med Wkly. 2020 Mar 23;150:w20229.

8 Lavina LWM, Fumeaux T. Intermediate care units: zwischen ICU und bettenstation. Schweiz Arztzeitg. 2017;98(25):802-3.

9 Plate JDJ, Leenen LPH, Houwert M, Hietbrink F. Utilisation of intermediate care units: a systematic review. Crit Care Res Pract. 2017; 2017:8038460.

10 Demoule A, Girou E, Richard JC, Taille S, Brochard L. Benefits and risks of success or failure of noninvasive ventilation. Intensive Care Med. 2006 Nov;32(11): 1756-65.

11 Rochwerg B, Brochard L, Elliott MW, Hess D, Hill NS, Nava S, et al. Official ERS/ATS clinical practice guidelines: noninvasive ventilation for acute respiratory failure. Eur Respir J. 2017 Aug;50(2):1602426.

12 Alhazzani W, Moller MH, Arabi YM, Loeb M, Gong MN, Fan E, et al. Surviving sepsis campaign: guidelines on the management of critically ill adults with Coronavirus Disease 2019 (COVID-19). Intensive Care Med. 2020 May; 46(5):854-87. 
13 Ferreyro BL, Angriman F, Munshi L, Del Sorbo L, Ferguson ND, Rochwerg B, et al. Association of noninvasive oxygenation strategies with all-cause mortality in adults with acute hypoxemic respiratory failure: a systematic review and meta-analysis. JAMA. 2020 Jul; 324(1):57-67.

14 Grieco DL, Menga LS, Raggi V, Bongiovanni F, Anzellotti GM, Tanzarella ES, et al. Physiological comparison of high-flow nasal cannula and helmet noninvasive ventilation in acute hypoxemic respiratory failure. Am J Respir Crit Care Med. 2020 Feb 1;201(3):303-12.

15 Patel BK, Kress JP, Hall JB. Alternatives to invasive ventilation in the COVID-19 pandemic. JAMA. 2020 Jul 7;324(1):43-4.

16 Gattinoni L, Chiumello D, Caironi P, Busana M, Romitti F, Brazzi L, et al. COVID-19 pneumonia: different respiratory treatments for different phenotypes? Intensive Care Med. 2020 Jun;46(6): 1099-1102.

17 Primmaz S, Le Terrier C, Suh N, Ventura F, Boroli F, Bendjelid K, et al. Preparedness and reorganization of care for coronavirus disease 2019 patients in a Swiss ICU: characteristics and outcomes of 129 patients. Crit Care Explor. 2020 Aug;2(8):e0173.
18 Frat JP, Thille AW, Mercat A, Girault C, Ragot S, Perbet S, et al. High-flow oxygen through nasal cannula in acute hypoxemic respiratory failure. N Engl J Med. 2015 Jun 4; 372(23):2185-96.

19 Stringhini S, Wisniak A, Piumatti G, Azman AS, Lauer SA, Baysson H, et al. Seroprevalence of anti-SARS-CoV-2 IgG antibodies in Geneva, Switzerland (SEROCoV-POP): a population-based study. Lancet. 2020 Aug; 396(10247):313-9.

20 Oranger M, Gonzalez-Bermejo J, DacostaNoble P, Llontop C, Guerder A, Trosini-Desert $\mathrm{V}$, et al. Continuous positive airway pressure to avoid intubation in SARS-CoV-2 pneumonia: a two-period retrospective casecontrol study. Eur Respir J. 2020 Aug 13; 56(2):2001692

21 Elharrar X, Trigui Y, Dols AM, Touchon F, Martinez S, Prud'homme E, et al. Use of prone positioning in nonintubated patients with COVID-19 and hypoxemic acute respiratory failure. JAMA. 2020 Jun 9;323(22):2336-8.
22 Sartini C, Tresoldi M, Scarpellini P, Tettamanti A, Carco F, Landoni G, et al. Respiratory parameters in patients with COVID-19 after using noninvasive ventilation in the prone position outside the intensive care unit. JAMA. 2020 Jun 9;323(22):2338-40.

23 Slessarev M, Cheng J, Ondrejicka M, Arntfield R. Critical care western research G. Patient self-proning with high-flow nasal cannula improves oxygenation in COVID-19 pneumonia. Can J Anaesth. 2020 Sep;67(9): 1288-90.

24 WHO Global. Clinical management of COVID-19: interim guidance; 27 May 2020. Available from: https://apps.who.int/iris/ handle/10665/332196.

25 Cai Q, Chen F, Wang T, Luo F, Liu X, Wu Q, et al. Obesity and COVID-19 severity in a designated hospital in Shenzhen, China. Diabetes Care. 2020 Jul;43(7):1392-8.

26 Tartof SY, Qian L, Hong V, Wei R, Nadjafi RF, Fischer H, et al. Obesity and mortality among patients diagnosed with COVID-19: results from an integrated health care organization. Ann Intern Med. 2020 Nov 17;173(10): 773-81. 